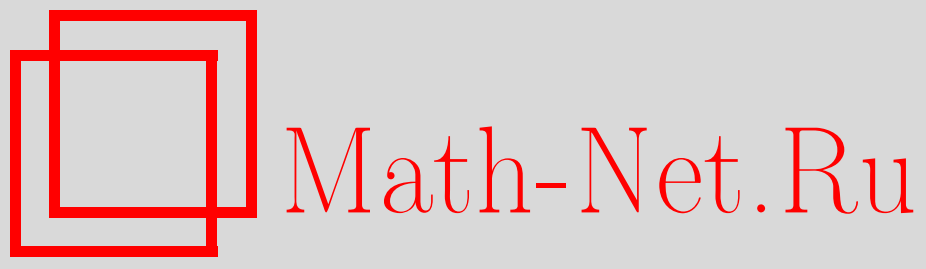

М. Л. Горбачук, Об аппроксимации решений операторных уравнений методом наименьших квадратов, Функи. анализ и его прил., 2005, том 39, выпуск 1, 85-90

DOI: https://doi.org/10.4213/faa34

Использование Общероссийского математического портала MathNet.Ru подразумевает, что вы прочитали и согласны с пользовательским соглашением

http://www. mathnet.ru/rus/agreement

Параметры загрузки:

IP : 54.92 .164 .108

26 апреля 2023 г., 13:25:22

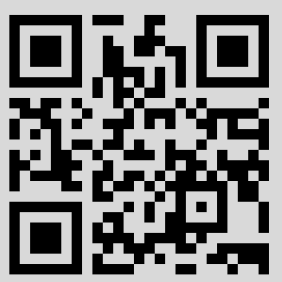


стоящими в $j$-й строке и $k$-м столбце $(k<j)$, где

$$
b_{p(j)}\left(\xi_{j}, \lambda\right)= \begin{cases}\lambda^{-m} a_{p(j)}\left(\xi_{j}\right), & \text { если } 1 \leqslant p(j) \leqslant n, \\ \lambda^{-(m-1)} a_{p(j)}\left(\xi_{j}\right), & \text { если } n+1 \leqslant p(j) \leqslant N .\end{cases}
$$

Матрица $Q_{\lambda}$ пределяется по формуле (10). Таким образом, в данном случае критерий гиперболичности легко проверяется.

\title{
ЛитерАТУРА
}

1. Вальтер Х.-О., Скубачевский А. Л. Труды ММО, 64, 3-53 (2003). 2. Хейл Дж. Теория функционально-дифференциальных уравнений. Мир, М., 1984. 3. Chow $S$. N., Diekmann O., Mallet-Paret J. Japan J. Appl. Math., 2, No. 2, 433-469 (1985). 4. Chow S. N., Walther H. O. Trans. Amer. Math. Soc., 307, No. 1, 127-142 (1988). 5. Walther H. O. Mem. Amer. Math. Soc., 402 (1989). 6. Diekmann O., van Gils S., Verduyn Lunel S., Walther H. O. Delay Equations. Functional, Complex, and Nonlinear Analysis. SpringerVerlag, New York, 1995. 7. Mallet-Paret J., Sell G. J. Differential Equations, 125, No. 2, 385-440 (1996).

Mathematisches Institut,

Поступило в редакцию Universitaet Giessen, Germany 28 июня 2004 г.

e-mail: Hans-Otto.Walther@math.uni-giessen.de

Московский авиационный институт

e-mail: skub@lector.ru

УДК 517.948

\section{Об аппроксимации решений операторных уравнений методом наименьших квадратов*}

\author{
(C) 2005. М. Л. ГОРБАЧУК
}

Пусть $\mathfrak{H}$ - сепарабельное гильбертово пространство над полем $\mathbb{C}$ комплексных чисел, $(\cdot, \cdot)$ и $\|\cdot\|$ - скалярное произведение и норма в нем, а $A-$ обратимый замкнутый линейный оператор с $\mathscr{D}\left(A^{-1}\right)=\mathfrak{H}(\mathscr{D}(\cdot)$ - область определения оператора).

Рассмотрим уравнение

$$
A u=f, \quad f \in \mathfrak{H},
$$

и будем искать его приближенное решение $u_{n}$ методом наименьших квадратов, т. е. в виде

$$
u_{n}=\sum_{k=1}^{n} \alpha_{k} e_{k}
$$

где $\left\{e_{k}\right\}_{k \in \mathbb{N}}$ - наперед выбранная линейно независимая система векторов из $\mathscr{D}(A)$ (так называемая координатная система), а $\alpha_{k} \in \mathbb{C}$ таковы, что величина

*Работа поддержана Совместным грантом CRDF и правительства Украины UM1-2567OD03. 
$R_{n}^{2}=\left\|A u_{n}-f\right\|^{2}$ принимает свое наименьшее значение. Числа $\alpha_{k}$ однозначно определяются системой уравнений

$$
\sum_{k=1}^{n} \alpha_{k}\left(A e_{k}, A e_{i}\right)=\left(f, A e_{i}\right), \quad i=1, \ldots, n .
$$

Если система $\left\{e_{k}\right\}_{k \in \mathbb{N}}$ полна в гильбертовом пространстве $\mathfrak{H}^{1}=\mathscr{D}(A)$ со скалярным произведением $(x, y)_{1}=(A x, A y)$, то, как известно (см., например, [1]),

$$
r_{n}=\left\|u-u_{n}\right\| \rightarrow 0, \quad R_{n}=\left\|A u_{n}-f\right\| \rightarrow 0 \quad \text { при } n \rightarrow \infty .
$$

Однако исследование зависимости асимптотического поведения величин $r_{n}$ и $R_{n}$ при $n \rightarrow \infty$ от выбора системы $\left\{e_{k}\right\}_{k \in \mathbb{N}}$, свойств параметров уравнения (1) и его решения $u$ оказалось довольно трудной задачей, которая в общем случае не решена до сих пор. Некоторые частные результаты для обыкновенных дифференциальных уравнений содержатся в [2, 3] и для уравнения (1) с самосопряженным положительно определенным оператором $A$ с дискретным спектром в [4]. Настоящая заметка посвящена решению указанной задачи в случае, когда $\left\{e_{k}\right\}_{k \in \mathbb{N}}$ - ортонормированный собственный базис самосопряженного положительно определенного оператора $B$, сходного с $A$, т. е. такого, что $\mathscr{D}(B)=\mathscr{D}(A)$. Представляемые здесь результаты носят завершенный характер в том смысле, что условия, гарантирующие определенный порядок стремления к нулю величин $r_{n}$ и $R_{n}$, сформулированные в терминах степени гладкости относительно оператора $B$ решения $u$, являются необходимыми и достаточными. Заметим еще, что вопрос зависимости степени гладкости решения $u$ от асимптотического поведения $r_{n}$ и $R_{n}$ на бесконечности (обратные теоремы) рассматривается впервые.

1. Всюду в дальнейшем предполагается, что

(i) оператор $A^{-1}$ компактный;

(ii) спектр $\lambda_{k}=\lambda_{k}(B)$ самосопряженного положительно определенного оператора $B$, сходного с $A$, простой;

(iii) в качестве координатной системы $\left\{e_{k}\right\}_{k \in \mathbb{N}}$ в методе наименьших квадратов выбран ортонормированный собственный базис оператора $B$.

Следующая теорема показывает, что величина $R_{n}=\left\|A u_{n}-f\right\|$ может стремиться к нулю на бесконечности произвольным образом.

Теорема 1. Пусть выполнены условия (i)-(iіi). Какова бъ ни была убъвающая числовая последовательность $\left\{\gamma_{n}\right\}_{n \in \mathbb{N}}, \gamma_{n}>0, \gamma_{n} \rightarrow 0(n \rightarrow \infty)$, существует вектор $f \in \mathfrak{H}$, такой, что

$$
\forall n \in \mathbb{N} \quad R_{n} \geqslant \gamma_{n}
$$

Естественно возникает вопрос, какими свойствами должно обладать решение $u$ уравнения (1) для того, чтобы величина $R_{n}$ имела определенный, наперед заданный порядок стремления к нулю. Ответ дает следующая (прямая)

Теорема 2. Предположим, что условия (i)-(iii) выполнены. Тогда для $\alpha \geqslant 0$

$$
u \in \mathscr{D}\left(B^{\alpha+1}\right) \Longrightarrow \lim _{n \rightarrow \infty} \lambda_{n+1}^{\alpha} R_{n}=0 .
$$


Поскольку $B$ - оператор, сходный с $A$, по теореме о замкнутом графике операторы $A B^{-1}$ и $B A^{-1}$ определены на всем $\mathfrak{H}$ и ограничены. Поэтому соотношение (2) эквивалентно утверждению

$$
u \in \mathscr{D}\left(B^{\alpha+1}\right) \Longrightarrow \lim _{n \rightarrow \infty} \lambda_{n+1}^{\alpha}\left\|B\left(u_{n}-u\right)\right\|=0 .
$$

Нетрудно привести пример, показывающий, что обратное к теореме 2 утверждение, вообще говоря, неверно, т. е. что равенство $\lim _{n \rightarrow \infty} \lambda_{n+1}^{\alpha}\left\|A u_{n}-f\right\|=0$ еще не гарантирует принадлежности $u \mathrm{~K} \mathscr{D}\left(B^{\alpha+1}\right)$. Зато имеет место

Теорема 3. B предположениях (i)-(iii) существует $\varepsilon>0$, такое, что

$$
\lim _{n \rightarrow \infty} \lambda_{n+1}^{\alpha+\varepsilon} R_{n}=0 \Longrightarrow u \in \mathscr{D}\left(B^{\alpha+1}\right) \text {. }
$$

Следующая теорема устанавливает связь между величиной $r_{n}=\left\|u-u_{n}\right\|$ и степенью гладкости относительно $B$ решения $u$ уравнения (1).

ТеОрема 4. Пусть выполняются условия (i)-(iii). Тогда

$$
\exists \varepsilon>0 \quad \lim _{n \rightarrow \infty} \lambda_{n+1}^{\alpha+\varepsilon} r_{n}=0 \Longrightarrow u \in \mathscr{D}\left(B^{\alpha}\right) .
$$

Eсли, кроме того, $\mathscr{D}\left(B^{2}\right)=\mathscr{D}\left(A^{*} A\right)$ u $\alpha \geqslant 2$, mо

$$
u \in \mathscr{D}\left(B^{\alpha}\right) \Longrightarrow \lim _{n \rightarrow \infty} \lambda_{n+1}^{\alpha} r_{n}=0 \text {. }
$$

Для самосопряженного положительно определенного $A$ и $\alpha=2$ второе утверждение теоремы содержится в [4]. Заметим также, что из условия $\mathscr{D}\left(B^{2}\right)=$ $\mathscr{D}\left(A^{*} A\right)$ следует, что $\mathscr{D}(B)=\mathscr{D}(A)$. Однако обратное, вообще говоря, не верно.

Обозначим через $C^{\infty}(B)$ множество всех бесконечно дифференцируемых векторов оператора $B$ :

$$
C^{\infty}(B)=\bigcap_{n \in \mathbb{N}_{0}} \mathscr{D}\left(B^{n}\right), \quad \mathbb{N}_{0}=\{0,1,2, \ldots\} .
$$

Из теорем 2-4 вытекает

СлЕДСТВИЕ 1. Имеют место соотношения

$$
u \in C^{\infty}(B) \Longleftrightarrow \forall \alpha>0 \lim _{n \rightarrow \infty} \lambda_{n+1}^{\alpha} R_{n}=0 \Longleftrightarrow \forall \alpha>0 \lim _{n \rightarrow \infty} \lambda_{n+1}^{\alpha} r_{n}=0 .
$$

2. Пусть $\left\{m_{n}\right\}_{n \in \mathbb{N}_{0}}$ - неубывающая последовательность положительных чисел (без ограничения общности можно считать, что $m_{0}=1$ ). Положим

$$
C_{\left\{m_{n}\right\}}(B)=\bigcup_{\alpha>0} C_{\alpha}\left\langle m_{n}\right\rangle(B), \quad C_{\left(m_{n}\right)}(B)=\bigcap_{\alpha>0} C_{\alpha}\left\langle m_{n}\right\rangle(B),
$$

где

$$
C_{\alpha}\left\langle m_{n}\right\rangle(B)=\left\{x \in C^{\infty}(B) \mid \exists c=c(x)>0:\left\|B^{k} f\right\| \leq c \alpha^{k} m_{k} \forall k \in \mathbb{N}_{0}\right\} .
$$

Следуя $[5,6]$, для вектора $x \in C_{\left\{m_{n}\right\}}(B)$ положим

$$
\sigma\left(x, m_{n}, B\right)=\inf \left\{\alpha: x \in C_{\alpha}\left\langle m_{n}\right\rangle(B)\right\}=\varlimsup_{n \rightarrow \infty}\left(\frac{\left\|B^{n} x\right\|}{m_{n}}\right)^{1 / n} .
$$

В конкретных ситуациях, когда $m_{n}=n$ ! и $m_{n} \equiv 1$, приходим к известным пространствам $C_{\{n !\}}(B), C_{(n !)}(B)$ и $C_{\{1\}}(B)$ аналитических [7], целых [8] и целых экспоненциального типа [9] векторов оператора $B$. В частности, если 
$\mathfrak{H}=L_{2}(a, b),-\infty<a<b<\infty, B=d / d x$ и $\mathscr{D}(B)-$ пространство Соболева $W_{2}^{1}(a, b)$, то $C^{(\infty)}(B)$ совпадает с множеством бесконечно дифференцируемых на $[a, b]$ функций, $C_{\{n !\}}(B)$ и $C_{(n !)}(B)$ - пространства аналитических на $[a, b]$ и соответственно целых функций и $C_{\{1\}}(B)$ совпадает с пространством целых функций экспоненциального типа. При $m_{n}=n^{n \beta}(\beta>1)$ получаем классы $\mathfrak{G}_{\{\beta\}}(B)=C_{\left\{n^{n \beta}\right\}}(B)$ и $\mathfrak{G}_{(\beta)}(B)=C_{\left(n^{n \beta}\right)}(B)$ бесконечно дифференцируемых функций Жевре типа Румье и Берлинга (см., например, [10]). В дальнейшем будем предполагать, что

и

$$
\lim _{n \rightarrow \infty} \sqrt[n]{m}_{n}=\infty
$$

$$
\forall h>1 \exists c=c(h)>0 \quad m_{n+1} \leqslant c h^{n} m_{n} .
$$

Так, например, при $\beta>0$ последовательности $m_{n}=n^{n \beta}$ и $m_{n}=(n !)^{\beta}$ удовлетворяют условиям (3) и (4).

Введем также функции

$$
\rho_{1}(\lambda)=\sup _{n \in \mathbb{N}_{0}} \frac{\lambda^{n}}{m_{n}}, \quad \rho_{2}(\lambda)=\sum_{n=0}^{\infty} \frac{\lambda^{n}}{m_{n}}, \quad \rho_{3}(\lambda)=\left(\sum_{n=0}^{\infty} \frac{\lambda^{2 n}}{m_{n}^{2}}\right)^{1 / 2} .
$$

При условии (3) все $\rho_{i}(\lambda)(i=1,2,3)$ положительны и монотонно стремятся к бесконечности при $\lambda \rightarrow \infty$; при этом $\rho_{2}(\lambda)$ допускает продолжение до целой функции. Если $\left\{m_{n}\right\}_{n \in \mathbb{N}_{0}}$ Удовлетворяет еще и (4), то

$$
\forall \alpha<1 \quad \exists c=c(\alpha) \quad \rho_{i}(\lambda) \geqslant c_{i} \lambda \rho_{i}(\alpha \lambda) .
$$

ТЕОРема 5. Пусть выполнены условия (i)-(iii) и последовательность $\left\{m_{n}\right\}_{n \in \mathbb{N}_{0}}$ удовлетворяет условиям (3) и (4). Тогда

$$
\begin{gathered}
u \in C_{\left\{m_{n}\right\}}(B), \sigma\left(u, m_{n}, B\right)=\sigma \Longleftrightarrow \\
\forall \varepsilon>0 \lim _{n \rightarrow \infty} \rho\left(\frac{\lambda_{n+1}}{\sigma+\varepsilon}\right) R_{n}=0, \varlimsup_{n \rightarrow \infty} \rho\left(\frac{\lambda_{n+1}}{\sigma-\varepsilon}\right) R_{n}=\infty ; \\
u \in C_{\left(m_{n}\right)}(B) \Longleftrightarrow \forall \varepsilon>0 \quad \lim _{n \rightarrow \infty} \rho\left(\frac{\lambda_{n+1}}{\varepsilon}\right) R_{n}=0,
\end{gathered}
$$

где $\rho(\lambda)$ - любал из функций $\rho_{i}(\lambda)$.

Заметим, что в теореме $5 R_{n}$ можно заменить на $r_{n}$.

ЗАмЕЧАниЕ. Если в качестве приближенного решения уравнения (1) взять вектор $u_{n}$, на котором функция $F(u)=\|A u-f\|^{2}$ достигает своего минимума на подпространстве $\mathfrak{H}_{n}=\mathfrak{H}_{\lambda_{1}} \oplus \mathfrak{H}_{\lambda_{2}} \oplus \cdots \oplus \mathfrak{H}_{\lambda_{n}}\left(\mathfrak{H}_{\lambda_{k}}-\right.$ собственное подпространство оператора $B$, соответствующее собственному числу $\lambda_{k}$ ), то в формулировках всех теорем можно опустить предположение (ii) о простоте спектра оператора $B$.

3. Положим

$$
\begin{gathered}
\mathfrak{H}=L_{2}(0, \pi), \quad A=(-1)^{m} \frac{d^{2 m}}{d t^{2 m}}+\sum_{k=0}^{2 m-1} p_{k}(t) \frac{d^{k}}{d t^{k}}, \\
\mathscr{D}(A)=\left\{v(\cdot) \in W_{2}^{2 m}[0, \pi] \mid v^{(2 k)}(0)=v^{(2 k)}(\pi)=0, k=0, \ldots, m-1\right\},
\end{gathered}
$$

где $p_{k}(\cdot) \in C[0, \pi]$. Предположим также, что уравнение $A v=0$ имеет только тривиальное решение. 
Оператор $B$ определим формулой

$$
B=(-1)^{m} \frac{d^{2 m}}{d t^{2 m}}, \quad \mathscr{D}(B)=\mathscr{D}(A) .
$$

Этот оператор является самосопряженным и положительно определен, его спектр $\left\{\lambda_{k}=k^{2 m}\right\}_{k \in \mathbb{N}}$ дискретный и простой, а функции $\sqrt{2 / \pi} \sin k t$ образуют собственный ортонормированный базис оператора $B$ в $L_{2}(0, \pi)$.

Нетрудно показать, что при дополнительных условиях на $p_{k}(t)$, а именно

$$
p_{k} \in C^{2 m j}[0, \pi]
$$

и

$$
\begin{array}{rll}
p_{k}^{(2 r-1)}(0)=p_{k}^{(2 r-1)}(\pi)=0 & \text { при четном } k & (1 \leqslant r \leqslant m j), \\
p_{k}^{(2 r)}(0)=p_{k}^{(2 r)}(\pi)=0 & \text { при нечетном } k & (0 \leqslant r \leqslant(m-1) j),
\end{array}
$$

справедливо равенство

$$
\mathscr{D}\left(A^{j+1}\right)=\mathscr{D}\left(B^{j+1}\right) .
$$

На основании теоремы 2 приходим к следующему выводу.

ПрЕДЛОЖЕНИЕ 1. Если коэффициенты $p_{k}(t)$ удовлетворяют условиям (6)(8), $a$

$$
f(\cdot) \in C^{2 m j}[0, \pi] \quad u \quad f^{(2 k)}(0)=f^{(2 k)}(\pi)=0 \quad(k=0,1, \ldots, m j-1),
$$

mo

$$
(n+1)^{2 m j}\left\|A u_{n}-f\right\|_{L_{2}(0, \pi)} \rightarrow 0 \quad \text { npu } n \rightarrow \infty,
$$

или, что эквивалентно

$$
(n+1)^{2 m j}\left\|u_{n}-u\right\|_{W_{2}^{2 m}[0, \pi]} \rightarrow 0 \quad(n \rightarrow \infty) .
$$

Заметим, что в случае, когда $m=1, j=1$, соотношение (10) было установлено в [2].

Из следствия 1 и теоремы 5 непосредственно вытекает

ПРЕДЛОЖЕНИЕ 2. Предположим, что $p_{k}(\cdot)$ принадлежат $C^{\infty}[0, \pi]$ и удовлетворяют условиям (6), (7) при любом $r \in \mathbb{N}_{0}$. Тогда включение $f(\cdot) \in$ $C^{\infty}[0, \pi]$ и выполнение условия (9) при $k \in \mathbb{N}_{0}$ эквивалентны утверждению

$$
\forall \alpha>0 n^{\alpha}\left\|u_{n}-u\right\|_{W_{2}^{2 m}[0, \pi]} \rightarrow 0 \quad(n \rightarrow \infty) .
$$

Если вдобавок функиии $p_{k}(t)$ аналитичны на $[0, \pi]$, то утвержсдение

$$
\exists \alpha>0 \quad e^{\alpha n}\left\|u_{n}-u\right\|_{W_{2}^{2 m}[0, \pi]} \rightarrow 0 \quad(n \rightarrow \infty)
$$

эквивалентно аналитичности функиии $f(t)$ на $[0, \pi]$ и условию (9) для любого $k \in \mathbb{N}_{0}$. Если же $p_{k}(t)$ - целые функиии, то, для того чтобы было справедливо утверждение

$$
\forall \alpha>0 \quad e^{\alpha n}\left\|u_{n}-u\right\|_{W_{2}^{2 m}[0, \pi]} \rightarrow 0 \quad(n \rightarrow \infty),
$$

необходимо и достаточно, чтобы функция $f(t)$ была целой и удовлетворяла условию (9) для произвольного $k \in \mathbb{N}_{0}$.

Заметим, что в предложении 2 величина $\left\|u_{n}-u\right\|_{W_{2}^{2 m}[0, \pi]}$ может быть заменена на $\left\|A u_{n}-f\right\|_{L_{2}^{2 m}[0, \pi]}$. 


\title{
ЛитеРАТУРА
}

1. Михлин C. Г. Вариационные методы в математической физике. Наука, М., 1970. 2. Крылов Н. М. Избранные труды, в 3-х т. Изд-во АН УССР, Киев, т. 3, 1961. 3. Лучка A. Ю., Лучка Т. Ф. Возникновение и развитие прямых методов математической физики. Наукова думка, Киев, 1985. 4. Джишкариани А. В. Ж. вычисл. матем. и матем. физ., 8, №5, 1110-1116 (1968). 5. Горбачук М. Л., Горбачук В. И. УМН, 48, вып. 4, 180 (1993). 6. Горбачук M. Л. Функц. анализ и его прил., 37, вып. 1, 75-78 (2002). 7. Nelson E. Ann. Math., 70, No. 3, 572-615 (1959). 8. Goodman R. Trans. Amer. Math. Soc., 143, 55-76 (1969). 9. Радыно Я. В. Докл. АН БССР, 27, № 9, 791-793 (1983). 10. Lions J. L., Magenes E. Problèmes aux limites non homogènes et applications, Vol. 3, Dunod, Paris, 1970.

Институт математики НАН Украины e-mail: imath@horbach.kiev.ua

Поступило в редакцию 16 мая 2003 г.

УДК $512.7+517.55$

\section{Новые примеры поверхностей в $\mathbb{C P}^{3}$, гиперболических по Кобаяши*}

\author{
(c) 2005. М. ЗАЙДЕНБЕРГ, Б. ШИФФМАН
}

Клеменс [2] показал, что очень общая ${ }^{1)}$ поверхность $X_{d}$ степени $d \geqslant 5$ в $\mathbb{C P}^{3}$ не содержит рациональных кривых. Ксю [10] доказал, что на $X_{d}$ также нет эллиптических кривых (и, стало быть, на $X_{d}$ нет кривых рода $<2$ ), т. е. $X_{d}$ алгебраически гиперболична. Согласно гипотезе Кобаяши, поверхность $X_{d}$ должна быть гиперболичной по Кобаяши и, следовательно, не должна содержать непостоянных целых кривых $\mathbb{C} \rightarrow X_{d}$. Известно [11], что это свойство открыто в хаусдорфовой топологии на проективном пространстве всех поверхностей данной степени $d$ и, более того, оно выполняется для очень общей поверхности степени $\geqslant 21$ в $\mathbb{C P}^{3}[3,6]$.

Примеры гиперболических поверхностей в $\mathbb{C P}^{3}$ строились неоднократно; см. ссылки в наших предыдущих работах $[8,9]$, где также даны такие примеры. На сегодня минимальная степень в известных примерах равна 8; первое семейство примеров степени 8 в $\mathbb{C P}^{3}$ было обнаружено Фуджимото [5] и независимо Дювалем [4]. В [9] мы воспользовались методом деформаций для построения нового примера гиперболической октики в $\mathbb{C P}^{3}$. Здесь мы предлагаем упрощенный вариант нашего метода для построения гиперболической октики в $\mathbb{C P}^{3}$, которая является деформацией объединения двух общих конусов в $\mathbb{C P}^{3}$ степени 4 . В действительности так строятся примеры любой степени, начиная с 8.

В силу наблюдения Мамфорда и Богомолова, доказанного в [7], любая К3-поверхность содержит рациональные и эллиптические кривые. В частности, такие кривые имеются на любой гладкой поверхности в $\mathbb{C P}^{3}$ степени $\leqslant 4$. Рациональную кривую на общей квартике в $\mathbb{C P}^{3}$ дает ее сечение трикасательной плоско-

* Исследования первого автора частично поддержаны NSF, грант DMS-0100474.

1) То есть принадлежащая в пространстве параметров дополнению к счетному объединению собственных алгебраических подмножеств. 